\title{
A novel concept of equilibrium over a network
}

\author{
Basilio Gentile, Dario Paccagnan, Bolutife Ogunsola, and John Lygeros
}

\begin{abstract}
We define and analyze a novel concept of equilibrium over a network, which we refer to as location equilibrium. Its applications include area coverage for taxi drivers, human migration and task assignment for a server network. In particular, we show that a specific instance of the location equilibrium problem is equivalent to the Wardrop equilibrium problem on a specific network. Further, we show that finding a location equilibrium is equivalent to solving a variational inequality with an operator which is in general not monotone. Based on the relation to the Wardrop equilibrium, we propose the use of the extragradient algorithm and show its convergence to a specific location equilibrium. The findings are applied to a numerical study of area coverage for taxi drivers in Hong Kong.
\end{abstract}

\section{INTRODUCTION}

In recent years there has been an increasing interest in modeling agents that interact through a graph. Network games emerged as a modeling language to describe noncooperative interactions between agents, where their mutual influence is captured by an underlying network; the works [1], [2] address the problem for finite strategy space and [3], [4] for continuous strategy space. Applications of this framework include social networks [4] and economic interactions [5, Section 1.4.3], traffic networks [6] and task allocation problems [7]. While in network games each node represents an agent and an edge connecting node $i$ to node $j$ indicates that the choice of agent $i$ influences the utility of agent $j$, our framework departs significantly from this. More precisely, given an underlying graph, we consider a fixed number of agents that need to distribute themselves over its nodes. The utility that each agent perceives by selecting a certain node depends on the total number of agents who select that same node. Each agent can possibly improve his utility by moving to a neighboring node at the expense of a fixed movement cost. Within this framework, we define a location equilibrium as a distribution of agents where none of them has any incentive to move to any neighboring node.

An important feature of our setup is that each agent possesses an infinitesimal mass, which has a negligible influence on the utility of the nodes. This assumption is the same that motivates the concept of Wardrop equilibrium in a road network [8]. It allows us to define a location equilibrium in terms of aggregate mass distribution rather than considering the strategy of each specific agent. Indeed the two equilibrium concepts have more in common: in

This work was supported by the Swiss Competence Centers for Energy Research FEEB\&D, by the European Commission project DYMASOS (FP7-ICT 611281), and by the SNSF grant number P1EZP2 172122. B. Gentile, D. Paccagnan, B. Ogunsola and J. Lygeros are with the Automatic Control Laboratory, ETH Zürich, Switzerland. Email: \{gentileb,dariop,lygeros\}@control.ee.ethz.ch and bogunsola@student.ethz.ch.
Section III we show that the Wardrop equilibrium of a parallel road network coincides with the location equilibrium for a complete graph with zero movement costs. Nonetheless, we argue that it is not possible in general to write one equilibrium as a specific instance of the other or viceversa.

The concept of equilibrium that resembles the most the location equilibrium here introduced is the migration equilibrium of Nagurney (see [9] and references therein). In [9] an equilibrium model of human migration is presented for a network with zero movement costs and utilities which depend on the mass distribution in the entire network. The work [10] builds upon [9] to account for positive movement costs and characterizes the migration equilibrium as the solution of an optimization program for the case of linear separable costs. A more general migration equilibrium model is given in [11] and [12] where the movements costs are not constant but rather functions of the movements across the network, and where agents are divided into different classes.

These two features are not present in our model, which on the other hand extends the works [9]-[12] in that we relax the assumption of "no repeated migration", which imposes that each agent can move at most once with respect to an initial agents configuration. Our main contribution consists in characterizing the location equilibrium as the solution of a variational inequality, which is in general not monotone, and thus difficult to solve. We observe that such variational inequality cannot be reduced in general to an optimization program. We further contribute by proposing an algorithm that is capable of finding one such equilibrium.

For the sake of completeness, we note that other works as [13], [14], [15] have built upon [9]-[12], but the setups and the results differ from those here presented.

Finally, the theoretical findings of this paper are used to perform a numerical study of area coverage for taxi drivers in the city of Hong Kong. The network data, customers spatial distribution and trip costs are taken from [16], while the utility functions are derived based on [17].

Organization: The paper unfolds as follows: Section II introduces the location equilibrium. Section III characterizes the location equilibria as solution set of a variational inequality, shows that such set is in general not convex and draws a connection with the Wardrop equilibrium. Section IV proposes the use of the extragradient algorithm to achieve a location equilibrium. Section $\mathrm{V}$ performs a numerical study of taxi area coverage and Section VI draws conclusions and future research directions.

Notation: We denote with $I_{n} \in \mathbb{R}^{n \times n}$ the identity matrix, with $\mathbb{1}_{n} \in \mathbb{R}^{n}$ the vector of unit entries and with $\mathbb{O}_{n} \in \mathbb{R}^{n}$ the vector of zero entries. Given $x \in \mathbb{R}^{n}$ or $A \in \mathbb{R}^{n \times m}$, 
$x>0$ or $A>0$ are meant to be entry-wise inequalities. Given $n$ real numbers $x_{i},\left[x_{1} ; \ldots ; x_{N}\right]:=\left[x_{i}\right]_{i=1}^{N} \in \mathbb{R}^{n}$.

\section{THE LOCATION EQUILIBRIUM}

We consider a directed weighted graph $G=(V, E, C)$, where $V=\{1, \ldots, n\}$ is the node set, $E \subseteq V \times V$ is the set of directed edges, and $C=\left\{c_{i, j}\right\}_{(i, j) \in E}$ is the set of non-negative weights. We assume that $(i, i) \in E$ and $c_{i i}=0$ for all $i \in\{1, \ldots, n\}$. Moreover, consider $n$ utility functions $\left\{u_{i}: \mathbb{R}_{\geq 0} \rightarrow \mathbb{R}\right\}_{i=1}^{n}$ and a constant $\gamma>0$ which we refer to as total mass. Given such setup, we intend to spread a total mass $\gamma$ over the nodes to reach an equilibrium, according to the following definition.

Definition 1 (Location equilibrium). A vector $x \in \mathbb{R}_{\geq 0}^{n}$ is a location equilibrium if $\sum_{i=1}^{n} x_{i}=\gamma$ and for each $i \in$ $\{1, \ldots, n\}$ such that $x_{i}>0$ it holds

$$
u_{i}\left(x_{i}\right)+c_{i j} \geq u_{j}\left(x_{j}\right), \quad \forall j \in \mathcal{N}_{i}^{\text {out }} .
$$

The quantity $x_{i}$ is the mass at node $i$ which enjoys utility $u_{i}\left(x_{i}\right)$. The weight $c_{i j}$ is to be interpreted as the cost of a movement from $i$ to $j$. In words, a mass distribution $x$ which sums to $\gamma$ is a location equilibrium if each node $i$ with nonzero mass $x_{i}$ sees no attractive neighbor; a node $j \in \mathcal{N}_{i}^{\text {out }}$ is an attractive neighbor for $i$ if the marginal utility that an infinitesimal amount of mass would encounter by moving from $i$ to $j$ is larger than the movement cost $c_{i j}$. Clearly, if $j$ is attractive for $i$ then $i$ is not attractive for $j$.

We conveniently define the simplex set as

$$
\mathcal{S}:=\left\{x \in \mathbb{R}_{\geq 0}^{n} \text { s.t. } \sum_{i=1}^{n} x_{i}=\gamma\right\} \subset \mathbb{R}^{n} .
$$

and the set of out-neighbors of $i$ as $\mathcal{N}_{i}^{\text {out }}:=\{j \in$ $\{1, \ldots, n\}$ s.t. $(i, j) \in E\}$. Moreover, given a distribution $x \in \mathcal{S}$, for each node $i$ such that $x_{i}>0$ we define the envy set of $i$ as

$$
\mathcal{E}_{i}^{\text {out }}(x):=\left\{j \in \mathcal{N}_{i}^{\text {out }} \text { s.t. } u_{i}\left(x_{i}\right)+c_{i j}<u_{j}\left(x_{j}\right)\right\} \subseteq \mathcal{N}_{i}^{\text {out }} .
$$

If instead $x_{i}=0$ we define $\mathcal{E}_{i}^{\text {out }}(x)=\emptyset$. For any $i \in$ $\{1, \ldots, n\}$, we define $\mathcal{E}_{i}^{\text {in }}(x)=\left\{j \in \mathcal{N}_{i}^{\text {in }}\right.$ s.t. $\left.i \in \mathcal{E}_{j}^{\text {out }}\right\}$. Using this notation

$$
\begin{aligned}
& \bar{x} \text { is a location equilibrium } \Leftrightarrow \\
& \bar{x} \in \mathcal{S} \text { and } \mathcal{E}_{i}^{\text {out }}(\bar{x})=\emptyset, \forall i \in\{1, \ldots, n\} .
\end{aligned}
$$

Definition 2 (Envy graph). Given $x \in \mathcal{S}$ and $0<\tau<1$, we define the envy graph $G^{\text {envy }}(x)$ as the directed weighted $\operatorname{graph}(V, E(x) \subseteq E, C(x))$ with

- $(i, j) \in E(x)$ if and only if $i=j$ or $j \in \mathcal{E}_{i}^{\text {out }}(x)$;

- $C(x)$ is such that, for all $i \in\{1, \ldots, n\}$

- if $\mathcal{E}_{i}^{\text {out }}(x)=\emptyset$ then $c_{i i}=1$, otherwise $c_{i i}=1-\tau$;

- for all $j \neq i$, if $j \in \mathcal{E}_{i}^{\text {out }}(x)$ then $c_{i j}=\tau /\left|\mathcal{E}_{i}^{\text {out }}(x)\right|$, otherwise $c_{i j}=0$.

We also introduce the weighted adjacency matrix $A(x)$ relative to $G^{\text {envy }}(x)$ as in [18]. Note that for all $x \in \mathcal{S}$, the adjacency matrix is row-stochastic, i.e. $A(x) \mathbb{1}_{n}=\mathbb{1}_{n}$.
By construction, the envy-graph $G^{\text {envy }}(x)$ has no edges (other than the self-loops) if and only $x$ is a location equilibrium. If instead $x$ is not a location equilibrium, then $G^{\text {envy }}(x)$ has a self-loop at every node and an edge from $i$ to $j \neq i$ if $i$ is envious of $j$, i.e. if $u_{i}\left(x_{i}\right)+c_{i j}<u_{j}\left(x_{j}\right)$.

Lemma 1. Given $x \in \mathcal{S}$, there exists $i \in\{1, \ldots, n\}$ such that $\mathcal{E}_{i}^{\text {out }}(x)=\emptyset$.

Proof. We conduct the proof by contradiction. Assume that $\mathcal{E}_{i}^{\text {out }}(x) \neq \emptyset$ for each node $i \in\{1, \ldots, n\}$. Then each node in $G^{\text {envy }}(x)$ has at least one out-going edge, hence $G^{\text {envy }}(x)$ contains a cycle. Without loss of generality, we assume that the vertexes in this cycle are $(1, \ldots, m)$. Then

$$
\begin{aligned}
& u_{i}\left(x_{i}\right)-u_{i+1}\left(x_{i+1}\right)+c_{i, i+1}<0, i=\{1, \ldots, m-1\}, \\
& u_{m}\left(x_{m}\right)-u_{1}\left(x_{1}\right)+c_{m, 1}<0 .
\end{aligned}
$$

Summing up the $m$ equations above results in $c_{12}+\cdots+$ $c_{m 1}<0$, which is a contradiction.

\section{REFORMULATION AS VARIATIONAL INEQUALITY}

\section{AND CONNECTION WITH WARDROP EQUILIBRIUM}

We show that the set of location equilibria coincides with the solutions of a certain variational inequality.

Definition 3 (Variational inequality). Consider a set $\mathcal{K} \subseteq \mathbb{R}^{n}$ and an operator $F: \mathcal{K} \rightarrow \mathbb{R}^{n}$. A point $\bar{x} \in \mathcal{K}$ is a solution of the variational inequality $\operatorname{VI}(\mathcal{K}, F)$ if

$$
F(\bar{x})^{\top}(x-\bar{x}) \geq 0, \quad \forall x \in \mathcal{K} .
$$

Let us define the operator $F: \mathcal{S} \rightarrow \mathbb{R}_{\geq 0}^{n}$ as follows

$$
\begin{aligned}
& F(x)=\left[F_{i}(x)\right]_{i=1}^{n}, \\
& F_{i}(x)=\max _{j \in \mathcal{N}_{i}^{\text {out }} \cup\{i\}}\left(u_{j}\left(x_{j}\right)-u_{i}\left(x_{i}\right)-c_{i j}\right),
\end{aligned}
$$

where we remind that $(i, i) \in E$ and $c_{i i}=0$ for all $i \in$ $\{1, \ldots, n\}$. As a consequence, for all $i \in\{1, \ldots, n\}$ it holds $F_{i}(x) \geq 0$ and $F_{i}(x)=0$ if and only if $i$ does not envy any of its neighbors, i.e. $\mathcal{E}_{i}^{\text {out }}(x)=\emptyset$.

Theorem 1. A vector $\bar{x} \in \mathbb{R}^{n}$ is a location equilibrium if and only if it is a solution of $\operatorname{VI}(\mathcal{S}, F)$.

Proof. Take $\bar{x}$ location equilibrium. By Definition 1 , for each $i \in\{1, \ldots, n\}$ at least one of the following holds

$$
\begin{aligned}
& \bar{x}_{i}=0, \text { or } \\
& u_{i}\left(\bar{x}_{i}\right)+c_{i j} \geq u_{j}\left(\bar{x}_{j}\right), \quad \forall j \in \mathcal{N}_{i}^{\text {out }} .
\end{aligned}
$$

Condition (3a) implies

$$
\bar{x}_{i}=0 \Rightarrow F_{i}(\bar{x}) \cdot\left(x_{i}-\bar{x}_{i}\right)=\underbrace{F_{i}(\bar{x})}_{\geq 0} \cdot \underbrace{x_{i}}_{\geq 0} \geq 0, \forall x \in S .
$$

Condition (3b) implies

$$
F_{i}(\bar{x})=0 \Rightarrow F_{i}(\bar{x})\left(x_{i}-\bar{x}_{i}\right)=0, \forall x \in S .
$$

We can conclude that

$$
F(\bar{x})^{\top}(x-\bar{x})=\sum_{i=1}^{n} \underbrace{F_{i}(\bar{x})\left(x_{i}-\bar{x}_{i}\right)}_{\geq 0} \geq 0,
$$


hence $\bar{x}$ solves $\operatorname{VI}(\mathcal{S}, F)$.

Take $\bar{x}$ solving $\operatorname{VI}(\mathcal{S}, F)$. Since the set $\mathcal{S}$ satisfies Slater's constraint qualification, $\operatorname{VI}(\mathcal{S}, F)$ is equivalent to its $\mathrm{KKT}$ system, [5, Proposition 1.3.4a]:

$$
\begin{aligned}
& F(\bar{x})+\mu \mathbb{1}_{n}-\lambda=\mathbb{O}_{n} \\
& \bar{x} \in S \\
& \lambda \geq 0 \\
& \lambda^{\top} \bar{x}=0,
\end{aligned}
$$

where $\mu \in \mathbb{R}$ is the dual variable corresponding to the constraint $\mathbb{1}_{n}^{\top} \bar{x}=\gamma$ and $\lambda \in \mathbb{R}^{n}$ is the dual variable corresponding to the constraint $\bar{x} \geq 0$. Making use of (1) and substituting the expression of $\lambda$ from (4a) into (4c) and (4d) results in the system

$$
\begin{aligned}
& 0 \leq \mu \mathbb{1}_{n}+F(\bar{x}) \perp \bar{x} \geq 0, \\
& \mathbb{1}_{n}^{\top} \bar{x}=\gamma .
\end{aligned}
$$

Lemma 1 ensures the existence of $i \in\{1, \ldots, n\}$ such that $F_{i}(\bar{x})=0$. Then $\mu<0$ is not possible, otherwise the nonnegativity condition on $\mu \mathbb{1}_{n}+F(\bar{x})$ is violated. Moreover, since $F(\bar{x}) \geq 0, \mu>0$ is not possible, as this would imply $\bar{x}=\mathbb{O}_{n}$ thus violating $(5 \mathrm{~b})$. We can conclude that

$$
\begin{aligned}
& 0 \leq F(\bar{x}) \perp \bar{x} \geq 0, \\
& \mathbb{1}_{n}^{\top} \bar{x}=\gamma .
\end{aligned}
$$

Clearly $\bar{x} \in \mathcal{S}$ and

$$
\bar{x}_{i}>0 \underset{(6 \mathrm{a})}{\Rightarrow} F_{i}(\bar{x})=0 \Leftrightarrow \mathcal{E}_{i}^{\text {out }}(\bar{x})=\emptyset,
$$

hence $\bar{x}$ is a location equilibrium by Definition 1 .

Corollary 1. For any directed weighted graph $G=$ $(V, E, C)$, continuous utility functions $\left\{u_{i}: \mathbb{R}_{\geq 0} \rightarrow \mathbb{R}\right\}_{i=1}^{n}$ and total mass $\gamma>0$, there exists a location equilibrium. $\square$

Proof. By [5, Corollary 2.2.6], $\operatorname{VI}(\mathcal{S}, F)$ admits at least one solution because $\mathcal{S}$ is closed, convex and $F$ is continuous, as it is the maximum of continuous functions.

\section{Non-monotonicity of the operator $F$}

In this subsection we show that the operator $F$ is in general not monotone.

Definition 4 (Monotone operator). An operator $F: \mathcal{K} \subseteq$ $\mathbb{R}^{n} \rightarrow \mathbb{R}^{n}$ is monotone on the set $\mathcal{K}$ if

$$
(F(x)-F(y))^{\top}(x-y) \geq 0, \quad \forall x, y \in \mathcal{K} .
$$

Monotonicity of an operator plays a fundamental role in the study of the corresponding VI. In particular, it is a sufficient condition to guarantee convergence towards a solution of the VI of a plethora of algorithms, as described in [5, Chapter 12]. To study monotonicity of the operator $F$ in (2), we give an equivalent characterization of monotonicity.

Lemma 2. [19, Proposition 2.1] An operator $F$ is monotone on $S \subseteq \mathbb{R}^{n}$ if and only if for every $x \in S$ each subgradient $A \in \partial F(x)$ is positive semidefinite.

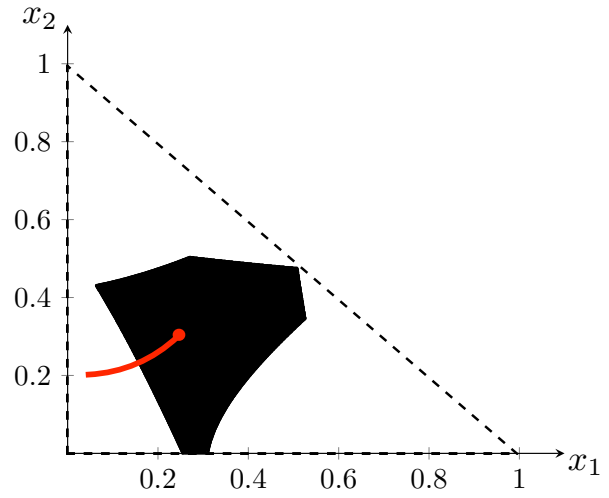

Fig. 1: We report in black the equilibrium set for Example 1, obtained by gridding the space and verifying for every point whether it satisfies Definition 1 or not. The set is visibly nonconvex. In dashed line we indicate the simplex boundary. We only report $x_{1}$ and $x_{2}$, as the third mass can be computed as $x_{3}=1-x_{2}-x_{3}$. In addition, the red line represents one trajectory of Algorithm 1, introduced in the following Section. The circle identifies the location equilibrium of $G_{0}$.

In the previous lemma we gave a characterization of monotonicity in terms of the subgradients of the operator $F$. A formal definition of these can be found in [19].

To show that the operator $F$ in (2) is not necessarily monotone, we give an example on a three-node network.

Example 1. Consider the complete three-node network described by the edge weights

$$
C=\left[\begin{array}{ccc}
0 & 0.4 & 0.9 \\
0.8 & 0 & 1 \\
0.3 & 0.6 & 0
\end{array}\right]
$$

and utility functions:

$$
\begin{aligned}
& u_{1}\left(x_{1}\right)=-2.5 x_{1}^{2}-0.51 x_{1}, \\
& u_{2}\left(x_{2}\right)=-3.75 x_{2}^{2}-0.33 x_{2}, \\
& u_{3}\left(x_{3}\right)=-0.5 x_{3}^{2}-0.42 x_{3}
\end{aligned}
$$

which are decreasing in $[0,1]$. The total mass is $\gamma=1$, that is, $\mathcal{S}=\left\{x \in \mathbb{R}_{\geq 0}^{3}\right.$ s.t. $\left.x_{1}+x_{2}+x_{3}=1\right\}$. One can easily verify that the operator $F$ is differentiable at the point $\hat{x}=[0.25,0.5,0.25]^{\top} \in \mathcal{S}$, so that the subgradient reduces to the gradient and reads as

$$
\nabla_{x} F(\hat{x})=\left[\begin{array}{ccc}
0 & 0 & 0 \\
-1.75 & 4.1 & 0 \\
0 & 0 & 0
\end{array}\right]
$$

The symmetric part of the matrix in (7) is indefinite, hence by Lemma $2 F$ is not monotone in $\mathcal{S}$.

The result in [5, Theorem 2.3.5] states that monotonicity of an operator guarantees convexity of the solution set of the corresponding VI. Figure 1 reports the set of location equilibria (which coincides with the set of solutions of the equivalent VI) for Example 1, which is not convex. 


\section{Connection with Wardrop equilibrium}

In this subsection we show that a specific instance of the location equilibrium in Definition 1 coincides with a specific instance of the Wardrop equilibrium for a traffic network [8], [20]. We note that such equivalence has already been shown in [9]. Nonetheless, stating the equivalence within the setup of this paper is useful to derive the results of the next Section IV.

Using the formalism of [6], we consider a road network with one origin $\mathcal{O}$, one destination $\mathcal{D}$ and $n$ parallel arcs joining $\mathcal{O}$ and $\mathcal{D}$ as by Figure 2. Each arc $i$ features a travel time equal to $T_{i}\left(x_{i}\right)$, which is a non-decreasing function of the amount of vehicles $x_{i} \geq 0$ on arc $i$. The total number of vehicles across all arcs is $\gamma$.

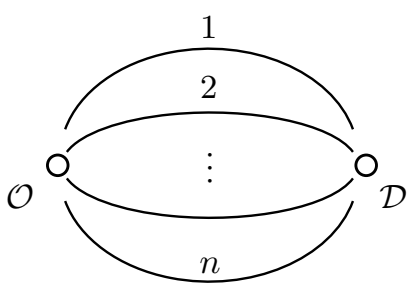

Fig. 2: Illustration of a parallel arc network.

In words [8], a configuration $\bar{x}=\left[\bar{x}_{i}\right]_{i=1}^{n}$ is a Wardrop equilibrium if and only if all the arcs used (i.e. where $\bar{x}_{i}>$ 0 ) have the same travel time $T_{\min }$ and such travel time is minimal. The work [6] formalized this concept by making use of a variational inequality.

Definition 5. A point $\bar{x} \in \mathcal{S}$ is a Wardrop equilibrium for the parallel arc network if it is a solution of $\operatorname{VI}(\mathcal{S}, T)$, with $T=\left[T_{i}\right]_{i=1}^{n}$.

Next let us consider the location equilibrium of Definition 1 over a complete graph, that is, a graph with $(i, j) \in E$ for all $i, j \in\{1, \ldots, n\}$. We consider a zero-cost graph, in the sense that $c_{i j}=0$ for all $i, j \in\{1, \ldots, n\}$. A complete zero-cost graph is denoted with $G_{0}=\left(V, V \times V, \mathbb{O}_{n^{2}}\right)$.

Proposition 1. A vector $\bar{x}$ is a Wardrop equilibrium of the parallel arc network with travel times $\left[T_{i}\right]_{i=1}^{n}$ and total number of vehicles $\gamma>0$ if and only if $\bar{x}$ is a location equilibrium for the complete zero-cost graph $G_{0}=\left(V, V \times V, \mathbb{O}_{n^{2}}\right)$ with utility functions $\left[u_{i}=-T_{i}\right]_{i=1}^{n}$ and total mass $\gamma$.

Proof. The point $\bar{x}$ is a solution of $\operatorname{VI}(\mathcal{S}, T)$ if and only if it solves its KKT system [5, Proposition 1.3.4a]

$$
\begin{aligned}
& 0 \leq \mu \mathbb{1}_{n}+T(\bar{x}) \perp \bar{x} \geq 0, \\
& \mathbb{1}_{n}^{\top} \bar{x}=\gamma,
\end{aligned}
$$

where $\mu \in \mathbb{R}$ is the dual variable corresponding to the constraint $\mathbb{1}_{n}^{\top} \bar{x}=\gamma$ and where we already performed the same simplification that led from (4) to (5). We now argue that it must hold

$$
\mu=-T_{\min }(\bar{x}):=-\min _{i \in\{1, \ldots, n\}} T_{i}\left(\bar{x}_{i}\right)
$$

with an argument very similar to the one we used after system (5). Indeed, if $\mu>-T_{\min }(\bar{x})$ then $\mu \mathbb{1}_{n}+T(\bar{x})>$ 0 , hence by the orthogonality condition $\bar{x}=0$ which violates (8b). If instead $\mu<-T_{\min }(\bar{x})$ then $\mu \mathbb{1}_{n}+T(\bar{x}) \geq 0$ does not hold. We can conclude that (8) reads as

$$
\begin{aligned}
& 0 \leq-T_{\min }(\bar{x}) \mathbb{1}_{n}+T(\bar{x}) \perp \bar{x} \geq 0, \\
& \mathbb{1}_{n}^{\top} \bar{x}=\gamma .
\end{aligned}
$$

Let us now substitute $T_{i}\left(\bar{x}_{i}\right)=-u_{i}\left(\bar{x}_{i}\right)$ which holds by assumption, to rewrite (9) as

$$
\begin{aligned}
& 0 \leq u_{\max }(\bar{x}) \mathbb{1}_{n}-u(\bar{x}) \perp \bar{x} \geq 0, \\
& \mathbb{1}_{n}^{\top} \bar{x}=\gamma .
\end{aligned}
$$

where we denoted $u(\bar{x})=\left[u_{i}\left(\bar{x}_{i}\right)\right]_{i=1}^{n}$ and $u_{\max }(\bar{x})=$ $\max _{i \in\{1, \ldots, n\}} u_{i}\left(\bar{x}_{i}\right)$. Then (10) is equivalent to (6), because for a complete zero-cost graph the $i^{\text {th }}$ component of $F$ in (2) reads

$$
F_{i}(\bar{x})=u_{\max }(\bar{x})-u_{i}\left(\bar{x}_{i}\right)
$$

We showed in the proof of Theorem 1 that (6) is equivalent to $\operatorname{VI}(\mathcal{S}, F)$, which by Theorem 1 coincides with the set of location equilibria.

We note that as a consequence of the above proof it holds

$$
\begin{aligned}
& \bar{x} \text { location equilibrium for } G_{0} \text { with utilities } u \Leftrightarrow \\
& \bar{x} \text { solves } \operatorname{VI}(\mathcal{S}, T) \Leftrightarrow \bar{x} \text { solves } \operatorname{VI}(\mathcal{S},-u) .
\end{aligned}
$$

We want to point out that it is generally not possible to express the Wardrop equilibrium of a generic road network as a location equilibrium, and it is not possible to express the location equilibrium with generic graph and costs as a Wardrop equilibrium.

\section{THE EXTRAGRADIENT ALGORITHM}

Lemma 3. Consider a weighted directed graph $G=$ $(V, E, C)$ and the corresponding complete zero-cost graph $G_{0}=\left(V, V \times V, \mathbb{O}_{n^{2}}\right)$. If $\bar{x}$ is a location equilibrium for $G_{0}$ then it is a location equilibrium for $G$.

\begin{tabular}{|c|c|}
\hline Algoritl & Extragradient algorithm \\
\hline Initializ & Set $k=0, x(0) \in S, \rho>0$. \\
\hline Iterate: & $\begin{array}{l}\tilde{x}(k+1)=\underset{\mathcal{S}}{\operatorname{Proj}}[x(k)+\rho u(x(k))], \\
x(k+1)=\underset{\mathcal{S}}{\operatorname{Proj}}[x(k)+\rho u(\tilde{x}(k))], \\
k=k+1 .\end{array}$ \\
\hline
\end{tabular}

Proof. If $\bar{x}$ is a location equilibrium for $G_{0}$ then for all $i \in$ $\{1, \ldots, n\}$

$$
\bar{x}_{i}=0 \text { or } u_{i}\left(\bar{x}_{i}\right) \geq u_{j}\left(\bar{x}_{j}\right), \forall j \in\{1, \ldots, n\} .
$$

Then $\bar{x}$ also satisfies for all $i \in\{1, \ldots, n\}$

$$
\bar{x}_{i}=0 \text { or } u_{i}\left(\bar{x}_{i}\right)+c_{i j} \geq u_{j}\left(\bar{x}_{j}\right), \forall j \in \mathcal{N}_{i}^{\text {out }} .
$$

where $\mathcal{N}_{i}^{\text {out }}$ is the set of out neighbors of $i$ in $G$.

We now introduce the extragradient algorithm [5, Algorithm 12.1.9] to achieve a location equilibrium for the complete zero-cost graph $G_{0}=\left(V, V \times V, \mathbb{O}_{n^{2}}\right)$. 
Note that Algorithm 1 has a centralized structure, because the projection onto the simplex set $\mathcal{S}$ can be computed only with information about all the agents.

Assumption 1. For each $i \in\{1, \ldots, n\}$, the utility function $u_{i}$ is non-increasing and Lipschitz with constant $L_{i}$.

Under Assumption 1 we define

$$
L_{\max }:=\max _{i \in\{1, \ldots, n\}} L_{i} .
$$

Proposition 2. Consider the weighted directed graph $G=$ $(V, E, C)$. If Assumption 1 holds and $\rho<1 / L_{\max }$, then Algorithm 1 converges to a location equilibrium for the graph $G$.

Proof. We just need to show that Algorithm 1 converges to a location equilibrium for $G_{0}$, because then Lemma 3 guarantees that this is a location equilibrium for $G$. To this end, we recall that the set of location equilibria for $G_{0}$ coincides with the solutions of $\operatorname{VI}(\mathcal{S},-u)$ as by (11). Since Algorithm 1 is the extragradient algorithm [5, Algorithm 12.1.9] applied to $\operatorname{VI}(\mathcal{S},-u)$, we verify the sufficient conditions of [5, Theorem 12.1.11] that guarantee its convergence to a solution of $\operatorname{VI}(\mathcal{S},-u)$. The set $\mathcal{S}$ is closed and convex. The operator $-u$ is monotone, because

$$
\begin{aligned}
& (-u(x)+u(y))^{\top}(x-y)= \\
& \sum_{i=1}^{n} \underbrace{\left(-u_{i}\left(x_{i}\right)+u_{i}\left(y_{i}\right)\right) \cdot\left(x_{i}-y_{i}\right)}_{\geq 0 \text { because } u_{i} \text { non-increasing }} \geq 0 .
\end{aligned}
$$

Having verified all the assumptions of [5, Theorem 12.1.11], we conclude the proof.

Remark. It is important to highlight that Algorithm 1 is centralized, because each step requires information of the utility values perceived at all nodes and because the projection onto the simplex set requires centralized computations. Further, note that Algorithm 1 converges to a location equilibrium of $G_{0}$, which in general might live in the interior of the equilibrium set of $G$. It is therefore possible that the proposed algorithm forces the agents towards an equilibrium of $G_{0}$, even if they already achieved a location equilibrium of $G$. The red trajectory in Figure 1 exemplifies this behavior.

\section{APPliCATION: AREA COVERAGE FOR TAXI DRIVERS}

In this section we apply the theory developed to the problem of area coverage for taxi drivers. Understanding the spatial and temporal behavior of taxi drivers has attracted the interest of the transportation community [21], [22], because it would allow to infer information for diverse scopes, ranging from land-use classification [23] to the analysis of collective behaviour of a city's population [24].

We focus on the urban area of Hong Kong, as the work [16] provides relevant data for our model. Indeed, [16] divides the area into $n=18$ neighborhoods, which in our setup are the graph nodes. We note that this analysis relies on the crucial assumption that a taxi driver in neighborhood $i$ enjoys the utility $u_{i}\left(x_{i}\right)$ without leaving the neighborhood during his trips. Our goal is to find an equilibrium distribution across the different neighborhoods of the urban area. We set an edge from $i$ to $j$ (and from $j$ to $i$ ) if only if the two neighborhoods are adjacent. The cost $c_{i j}$ is taken as the fuel cost of a trip from $i$ to $j$ according to [25] and $c_{i j}=c_{j i}$. The resulting graph is then undirected. The utility $u_{i}\left(x_{i}\right)$ is computed as follows

$$
u_{i}\left(x_{i}\right)=\alpha_{i} v_{i}\left(x_{i}\right)-\left(1-v_{i}\left(x_{i}\right)\right) \beta
$$

where $\alpha_{i}$ is the average profit per trip starting from location $i$ (which ranges from 30 to $140 \mathrm{HK} \$$ according to Figure 4 in [16]), $\beta=6.34 \mathrm{HK} \$$ is the operational cost of the of vacant taxi trips at the evening peak, which includes fuel costs, rental costs and the toll charges associated with the trips, taken from [16]. The quantity $v_{i}\left(x_{i}\right)$ is the matching function, which in our context represents the percentage of the time a taxi is occupied and according to [17, equation (1)] takes the form

$$
v_{i}\left(x_{i}\right)=1-\left(\frac{x_{i}}{1+x_{i}}\right)^{p_{i}}
$$

where $p_{i}>1$ is the number of passengers requesting a taxi at node $i$, which we take to be proportional to the values in Figure 3 of [16]. Note that $v_{i}(0)=1$ and $\lim _{x_{i} \rightarrow \infty} v_{i}\left(x_{i}\right)=$ 0 , as one would expect. Moreover, through simple algebraic computations $u_{i}\left(x_{i}\right)$ can be shown to be decreasing for $x_{i} \geq$ 0 and to have Lipschitz constant

$$
L_{i}=4\left(\alpha_{i}-\beta\right) p_{i} \frac{\left(p_{i}-1\right)^{p_{i}-1}}{\left(p_{i}+1\right)^{p_{i}+1}} .
$$

We run Algorithm 1 with stopping criterion $\| x(k+1)-$ $x(k) \| \leq 10^{-6}$ and we set $\rho=10^{-3}$, which is smaller than $1 / L_{\max }=1.7 \cdot 10^{-3}$, as required by Proposition 2 . Table I reports the number of iterations needed to reach convergence. We note that this number is high due to the small value of $\rho$ imposed by the theoretical bound of Proposition 2. For this reason we perform another simulation with the value $\rho=10^{-2}$, which provides no theoretical guarantees of convergence. Nonetheless, Algorithm 1 converges in 1000 different repetitions with random initial conditions. The number of iterations is also reported in Table I and it is substantially decreased compared to the previous choice of $\rho$. Figure 3 represents the equilibrium $\bar{x}$ obtained using Algorithm 1.

\begin{tabular}{ccc}
$\rho$ & Mean & Standard deviation \\
\hline $10^{-3}$ & 690.1 & 21.3 \\
\hline $10^{-2}$ & 79.2 & 2.5 \\
\hline
\end{tabular}

TABLE I: Number of iterations needed to reach convergence; we report mean and standard deviation for 100 repetitions of Algorithm 1, starting from random initial conditions on the simplex, for two different choices of $\rho$. Each iteration is counted twice, as it repeats twice the projection operation. 


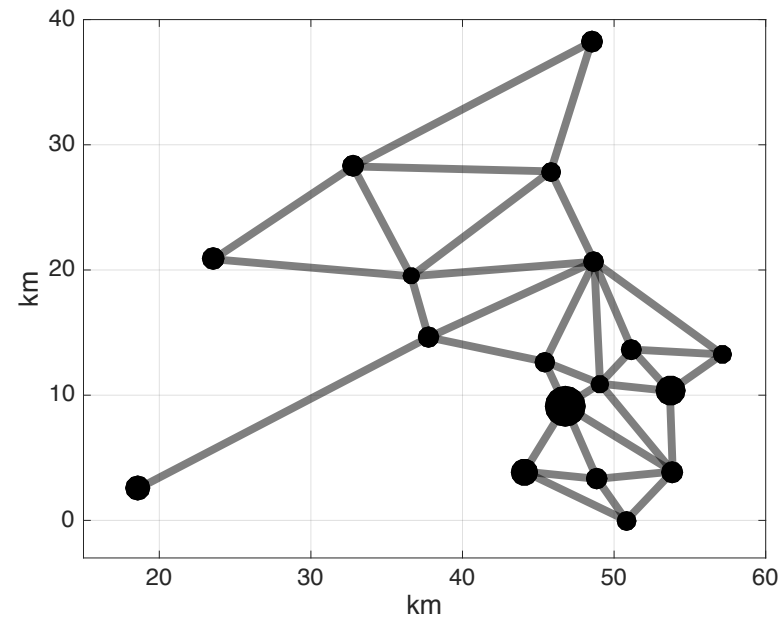

Fig. 3: The equilibrium $\bar{x}$ achieved by Algorithm 1 with initial condition $x(0)=\mathbb{1}_{n} / \gamma$. The size of node $i$ is proportional to its mass $x_{i}$. The length of an edge is proportional to the distance between the two neighborhoods it connects.

\section{CONCLUSIONS}

In this paper we defined a novel concept of equilibrium over a network and showed that the extragradient algorithm converges to one such equilibrium. Future research directions include extending the setup to allow for multiple classes of agents, studying the relation with the concept of Nash equilibrium and including time-varying movements costs.

At the end of Section IV we pointed out how the extragradient algorithm requires centralized computations and forces the agents to move even thought they are already at equilibrium. Future research aims at overcoming both these shortcomings.

\section{REFERENCES}

[1] A. Galeotti, S. Goyal, M. O. Jackson, F. Vega-Redondo, and L. Yariv, "Network games," The review of economic studies, vol. 77, no. 1, pp. 218-244, 2010.

[2] G. Szabó and G. Fath, "Evolutionary games on graphs," Physics reports, vol. 446, no. 4, pp. 97-216, 2007.

[3] S. Liang, P. Yi, and Y. Hong, "Distributed Nash equilibrium seeking for aggregative games with coupled constraints," arXiv preprint arXiv: 1609.02253, 2016.

[4] F. Parise, S. Grammatico, B. Gentile, and J. Lygeros, "Network aggregative games and distributed mean field control via consensus theory," arXiv preprint arXiv:1506.07719, 2015.

[5] F. Facchinei and J.-S. Pang, Finite-dimensional variational inequalities and complementarity problems. Springer, 2007

[6] S. Dafermos, "Traffic equilibrium and variational inequalities," Transportation science, vol. 14 , no. 1, pp. 42-54, 1980 .

[7] P. Yi and L. Pavel, "A distributed primal-dual algorithm for computation of generalized Nash equilibria with shared affine coupling constraints via operator splitting methods," arXiv preprint arXiv:1703.05388, 2017.

[8] J. G. Wardrop, "Some theoretical aspects of road traffic research," Proceedings of the institution of civil engineers, vol. 1, no. 3, pp. 325-362, 1952.

[9] A. Nagurney, "Migration equilibrium and variational inequalities," Economics letters, vol. 31, no. 1, pp. 109-112, 1989.

[10] — , "A network model of migration equilibrium with movement costs," Mathematical and Computer Modelling, vol. 13, no. 5, pp. 79-88, 1990
[11] A. Nagurney, J. Pan, and L. Zhao, "Human migration networks," European journal of operational research, vol. 59, no. 2, pp. 262274, 1992.

[12] - "Human migration networks with class transformations," in Structure and Change in the Space Economy. Springer, 1993, pp. 239-258.

[13] J. Pan and A. Nagurney, "Using markov chains to model human migration in a network equilibrium framework," Mathematical and computer modelling, vol. 19, no. 11, pp. 31-39, 1994.

[14] V. Kalashnikov, N. Kalashnykova, R. L. Rojas, M. M. Muños, C. Uranga, and A. L. Rojas, "Numerical experimentation with a human migration model," European Journal of Operational Research, vol. 189, no. 1, pp. 208-229, 2008.

[15] R. J. Cebula and R. K. Vedder, "A note on migration, economic opportunity, and the quality of life," Journal of Regional Science, vol. 13, no. 2, pp. 205-211, 1973.

[16] R. C. P. Wong, W. Y. Szeto, S. Wong, and H. Yang, "Modelling multiperiod customer-searching behaviour of taxi drivers," Transportmetrica B: Transport Dynamics, vol. 2, no. 1, pp. 40-59, 2014.

[17] N. Buchholz, "Spatial equilibrium, search frictions and efficient regulation in the taxi industry," Working paper, Tech. Rep., 2015.

[18] R. Olfati-Saber, J. A. Fax, and R. M. Murray, "Consensus and cooperation in networked multi-agent systems," Proceedings of the IEEE, vol. 95, no. 1, pp. 215-233, 2007.

[19] S. Schaible et al., "Generalized monotone nonsmooth maps," Journal of Convex Analysis, vol. 3, pp. 195-206, 1996.

[20] B. Gentile, F. Parise, D. Paccagnan, M. Kamgarpour, and J. Lygeros, "Nash and wardrop equilibria in aggregative games with coupling constraints," arXiv preprint arXiv:1702.08789, 2017.

[21] P. S. Castro, D. Zhang, and S. Li, Urban Traffic Modelling and Prediction Using Large Scale Taxi GPS Traces. Berlin, Heidelberg: Springer Berlin Heidelberg, 2012, pp. 57-72.

[22] B. Li, D. Zhang, L. Sun, C. Chen, S. Li, G. Qi, and Q. Yang, "Hunting or waiting? discovering passenger-finding strategies from a large-scale real-world taxi dataset," in Pervasive Computing and Communications Workshops (PERCOM Workshops), 2011 IEEE International Conference on. IEEE, 2011, pp. 63-68.

[23] G. Pan, G. Qi, Z. Wu, D. Zhang, and S. Li, "Land-use classification using taxi gps traces," IEEE Transactions on Intelligent Transportation Systems, vol. 14, no. 1, pp. 113-123, 2013.

[24] P. S. Castro, D. Zhang, C. Chen, S. Li, and G. Pan, "From taxi gps traces to social and community dynamics: A survey," ACM Computing Surveys (CSUR), vol. 46, no. 2, p. 17, 2013.

[25] (2017) Viamichelin. [Online]. Available: https://www.viamichelin.com 